\title{
Analysis on Computer Algorithm and Display Methods of Network Diagrams
}

\author{
Jin $\mathrm{He}^{1}$, Hongchong Zhang ${ }^{2}$ \\ ${ }^{1}$ School of Electrical Engineering, Chongqing Vocational Institute of Engineering , Chongqing, \\ 400037, China \\ ${ }^{2}$ Department of Party Committee Propaganda, Chongqing Vocational Institute of Engineering, \\ Chongqing, 400037, China
}

Key words: Analysis on network diagrams, Computer algorithm, Display methods, Analysis.

\begin{abstract}
With the rapid development of information technology, the combination of information technology with digital-related knowledge, and the widespread use of information technology in real life, many real problems need to be solved by building mathematical model, and the probability of solving some problems about discrete structure with mathematical technology becomes increasingly high. Under this context, the writer introduced the history of graph theory and the future development direction first, then analyzed the computer algorithm and display methods of network diagrams in detail, and put forward some methods of improving analysis on computer algorithm and display methods of network diagrams based on the actual situation and the future development direction.
\end{abstract}

\section{Introduction}

With the rapid development of information technology, the rate of using network diagrams in process flow, circuit network and communication network becomes higher and higher. Thus, the connection between computer technology and network diagram technology becomes closer. With network diagrams, the relations and structures of discrete elements in the original network can be analyzed quantitatively and qualitatively in a better way, and processed for optimization. On this basis, the shortest path and connectivity of the initial diagrams can be explored for optimization according to the vertex of weights and adjacency relationship of sides. Hence, it is of high use value. In view of this, the writer, focusing on analysis of the computer algorithm and display methods of network diagrams, made detailed analysis and discussions from three aspects, with an aim to improving people's knowledge thereabout, and then promoting people to apply it better, as below.

\section{History and Development of the Graph Theory}

The thought arisen from Chess in 1859 related to the graph theory, which is the origin of the graph theory. The most famous is the "Seven Bridges" put forward in East Prussia in the $18^{\text {th }}$ century. Through practices, it was found that many real problems can be solved in the best way by building mathematical model, specifically analyzing with lines and dots. Take the example of the "Seven Bridges". As shown in Figure 1, the two shoals in the rivers divided the land into four parts, which were marked with A, B, C and B respectively. The lands were connected via seven bridges. How can you walk all the bridges but each only once? Practices and experiments were carried out, but no solution was developed. Since there was no idea of solving problems with the graph theory, this problem became unsolvable. In 1936, Euler introduced related ideas on the graph theory while trying to solve this problem, and finally solved it by abstract analysis. He drew Figure 1(b) to abstract the problem of Figure 1(a). The dots in Figure 1(b) referred to the land parts, and the lines among the dots referred to the locations of bridges. It is thus clear that the path held only when the number of lines between every two dots was an even number. Since then, the idea of the graph theory has gradually been accepted. Over the past two hundred odd years since the emergence of the graph theory, the 
graph theory has gradually developed into an important component of solving real problems with mathematics. It is one of the important components of mathematics, and has gradually fused with other knowledge to a certain extent ${ }^{[1]}$ in its development process.

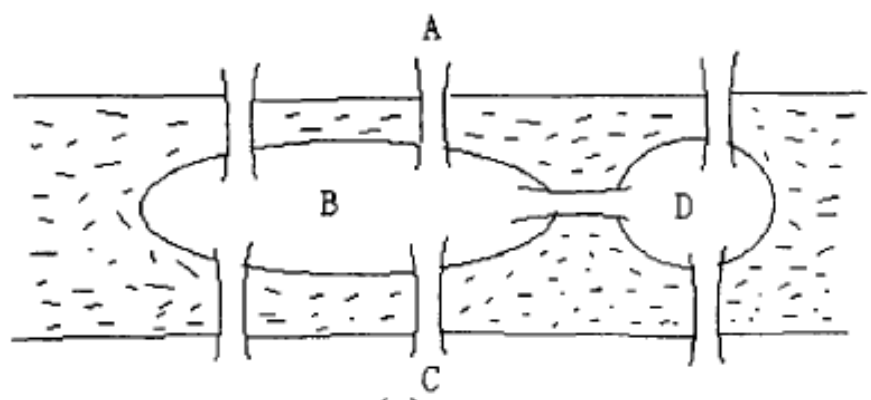

(a)

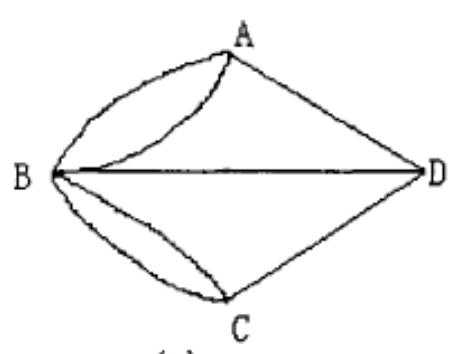

(b)

Fig. 1.

With the continuous development and advancement of computer in future, and to enhance the calculation capacity of computer, constant optimization and fusion of theoretical knowledge of mathematics should be made. For this purpose, the graph theory plays an important role. With the constant study by scientists, a great progress has been made. From the perspective of modern social environment, science and technology have become the main part of economic development, and computer technology has gradually become the core technology, which have great influence on both life and production of human. With the constant development of computer technology, the graph theory also develops constantly. Therefore, in the future development, the graph theory will be used more widely.

\section{Research on Computer Algorithm and Display Methods of Network Diagrams}

\section{Point Symbol Total Control Algorithm}

The research methodology on the best optimization measures of local problem is point symbol control algorithm. In the point symbol algorithm, the most advanced extended theory is point symbol total control algorithm. The greatest achievement of the point symbol total control algorithm is the realization of different research perspectives by expanding to realize an open field for the points that were originally put in a closed field. Furthermore, the upper and lower bounds of this research method have been constantly introduced on this basis, namely the minimum degree and maximum degree, and a construction method satisfying the next generation of network diagrams has been worked out. In the latest research, the main research direction is the reverse direction of $f[v]>1$ in the definition of the symbol control algorithm, and then to form a concrete research idea of reverse symbol total control algorithm, having increased the computation speed of network diagrams to a certain extent ${ }^{[2]}$.

\section{Edge Symbol Control Algorithm}

Edge symbol control algorithm and point symbol control algorithm have a lot in common in respect of the fundamental point, both of which are new type algorithms derived from symbol edge control. The emergence of edge symbol control algorithm has enriched and extended the algorithm of network diagram control to a large extent, and determined the boundary of general network diagram edge control algorithm with the concrete value of figures of some specific network diagram symbol edge control algorithms. As the research methods and perspectives in this area are constantly upgraded, in the latest research the value domain of function has been changed from [-1,1] into [-1.0.1] first, and a trimming control algorithm has been developed. It is more difficult, and so far no real achievements have been made. 


\section{Computer Display of Network Diagrams}

We usually use C language for programming when drawing network diagrams. On one hand, it is easy to program with $C$ language, and $C$ language is widely used; on the other hand, the codes written with $\mathrm{C}$ language occupy a small storage space of computer, and are executed at a high speed. Node and connecting line are parts of network diagrams, but node and connecting line are in a complicated mechanism relationship. According to the mathematical theory and knowledge, if a side is provided, it is bound to have two corresponding nodes ${ }^{[3]}$. Therefore, to draw a network diagram on a computer, corresponding nodes must be drawn first, and sidelines can be added according to the correlations between nodes. Specifically, the location of nodes should be determined first, and the coordinates $(\mathrm{x}, \mathrm{y})$ of connecting lines should be marked according to the coordinates of nodes, to draw a network diagram. It needs to be noted that in the case of drawing with $C$ language, physical coordinates should be referred to, namely taking the top left corner of the screen as the origin, marking the horizontal direction with $\mathrm{X}$ and the vertical direction with $\mathrm{Y}$, assigning the values below to be positive, as shown in Figure2. The first step is to confirm the coordinates (x,y) on the diagram, and both $\mathrm{X}$ and $\mathrm{Y}$ should be positive and have a specific value range. They are associated with the preset graphical modeling. In other words, they are related to the screen resolution. The relation expression of physical coordinates and user coordinates is as below:

$\mathrm{x} 1-\mathrm{x} 2$ refers to a numerical interval, and $\mathrm{X} 1-\mathrm{X} 2$ to a screen point interval, and then the relation expression of $\mathrm{x}$ and $\mathrm{X}$ is:

$$
\begin{array}{r}
X=\frac{X 2-X 1}{x 2-x 1} \cdot x+\frac{x 2 . X 1-x 1 . X 2}{x 2-x 1} \\
(x 1 \leq x \leq x 2, X 1 \leq X \leq X 2)
\end{array}
$$

For the direction of Y-axis:

y1-y2 refers to a numerical interval, and Y1-Y2 to a screen point interval, and then the relation expression of $\mathrm{y}$ and $\mathrm{Y}$ :

$$
\begin{array}{r}
Y=\frac{Y 2-Y 1}{y 2-y 1} \cdot y+\frac{Y 2 \cdot \mathrm{y} 2-\mathrm{Y} 1 \cdot y 1}{y 2-y 1} \\
(y 1 \leq \mathrm{y} \leq \mathrm{y} 2, \quad Y 1 \leq Y \leq Y 2)
\end{array}
$$

$\mathrm{X}, \mathrm{Y}$ should be rounded.

After the above steps are finished, it is needed to draw a side on the screen, determine the coordinates based on the two vertexes in correspondence with the side, and draw a straight line to link the two vertexes. This method is easy. Besides, there are many colors available in C language. Thus, user can use different colors to show the relationships of nodes ${ }^{[4]}$.

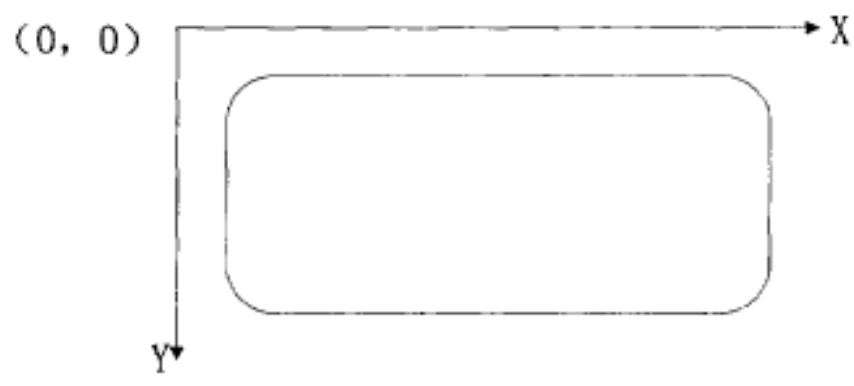

Fig. 2. Physical Coordinate System 


\section{Direction of Improvement of Computer Algorithm and Display Methods of Network Diagrams}

The shortest path question is one of the most classic questions on the network optimization theory and graph theory, and also the main direction of improvement of computer algorithm and display methods of network diagrams in future. With the rapid development of information technology and computer technology, we have entered the network era. In the network era, as the network becomes increasingly complicated, new requirements for computer algorithm are put forward, and more stress should be laid on instantaneity and speed in respect of the question of shortest path. How can the search range be constantly narrowed and the computation be simplified while accurate computation is guaranteed is the main research direction of optimization nowadays. With the constant development of computer data structure and other related technologies, and the combination of these technologies with traditional shortest path computation, a series acceleration techniques of shortest path computation emerged, mainly including hierarchical abstraction of network, improvement of search strategy, and improvement of algorithm running configuration, as below.

\section{Hierarchical Abstraction of Network}

Hierarchical strategy is an important way of thinking of human, and is widely used to solve problems with spatial features ${ }^{[5]}$. As to the optimization of network diagrams, the exploration of shortest path is the main research direction in future. The algorithm and computational complexity of shortest path are closely related to the density of related network size, and will increase in a non-linear way with the increase of the number $m$ of edges and the number $n$ of nodes in network. For the optimization of shortest path, one of the measures for simplifying the algorithm is to reduce order, and then select part of edge and node data to build a hierarchical network model, to simplify the topological relation of network. In the topology structure of each layer of network, the nodes at layer i+1 refer to the subset of layer i, and can be obtained via the network partition of the ith layer. In a two-level network layer, for computing the shortest path of the hierarchical topology of this layer, the initial point and local adjacent area should be expanded in the original network first, then skip to the superior network layer proceeds, and search should continue in the original network and adjacent area when the target $t$ is close until the target $t$ is reached. According to the analysis above, it can be seen that shortening search time can be realized by directly switching search to the superior network topology, which is also exactly the goal of hierarchical abstraction of network ${ }^{[6]}$.

\section{Improvement of Search Strategy}

In the traditional algorithm of shortest path, the blindness existing in search results in that there are a lot of nodes in the director opposite from the initial point to the target point $T$, and then causes the waste of search. Thus, in the traditional shortest path algorithm, the number of ergodic nodes is sure to be reduced by integrating heuristic information, and then shorten the search time. In concrete implementation, according to the hierarchical strategy, priority selection of the t artery or highway from the place of departure to the destination will be made; according to the direction strategy, relevant path leading to the destination will be selected; according to the greedy strategy, a straight path will be selected as far as possible. The guiding acceleration technology adopted in the existing researches on shortest path algorithm also was obtained by transformation according to the hierarchical strategy, direction strategy and greedy strategy of human thinking ${ }^{[7]}$.

\section{Improvement of Algorithm Running Configuration}

The running data of shortest path algorithm is of various structures, and each of which has their own features. The common structures include heap structure and bucket structure. For purpose of this paper, optimal exploration from the perspective of heap structure was carried out. The heap structure is categorized into binomial heap, binary heap and Fibonacci heap. For purpose of this paper, optimal exploration from the perspective of binary heap was carried out. The internal memory of binary heap is stored into a complete binary tree, of which every node satisfies the characteristics of its heap sequence. That's to say, the key values of nodes stored outside the root node should be larger than or 
equal to that stored in its father node. The basic operations are: encode $\mathrm{k}$ candidate nodes from top to bottom and from left to right to form a complete binary tree, scan from the last node to the root node one by one, realize that the currently scanned point is the exponential of the root node with related technology, and transform it into heap. Due to $K+n$, the time this computation takes is near $0(\operatorname{logn})^{[8]}$. Before algorithm iteration of the next step, certain structure maintenance is required. The main steps are deleting, inserting and modifying the minimum value. The foregoing statement shows that for sparse network, the adoption of heap structure can largely improve the performance of shortest path algorithm, while for complicated network diagrams, a bad result will be got if heap structure is adopted, namely efficiency reduction.

To draw network diagrams in the screen, related information of network diagrams should be input. The steps are as follows: input network diagrams - add a number of edges - add a number of vertexes — delete a number of edges—delete a number of vertexes—history query—-time query.

\section{Conclusion}

In the field of science, network diagrams are widely popular and attract a lot of attention for the superiority of its theory and its wide application range. For researches in this field, it is suggested to emphasize talent cultivation while persisting in scientific inquiry, and set rational and frontier research objectives. According to this paper, researches on point symbol total control algorithm, edge symbol control algorithm and computer display of network diagrams are mainly carried out. For future research, efforts can be made to realize the development and advancement of network diagrams from the aspects of hierarchical abstraction of network, improvement of search strategy and improvement of algorithm running configuration.

\section{Acknowledgments}

This paper belongs to the 2016 scientific and technological research program of Chongqing City Board of Education Committee, titled Researches on Key Network Technology of Large-scale Wireless Sensor for Water Environment Monitoring of the Three Gorges Region, (numbered KJ1603206), and organized by He Jin.

\section{References}

[1] Zhang Yanchao, Liu Jianzhuo, Sun Qiang, et al. Researches on Network Image Transmission Based on Windows CE. Proceedings of 2010 National Conference of China Communication Security. 2010, 41 (7): 294-299.

[1] Zhang Xiao. Researches on Clustering Method of High-dimensional Data Based on Super-network. Shandong Normal University,2015,21(9):20-24.

[2] Zhao Yiming, Lyu Penghui. Researches on Subject Knowledge Network (III) Structure, Features and Evolution of Co-word Network 1). Journal of the China Society for Scientific and Technical Information,2014,12(4):21-32.

[3] Dai Xiaofei. Enlightenment of Volunteer Service Mode of Reading Promotion Social Enterprise to Library-IN LIBRARY.The Library Journal of Henan,2015,36(10):96-98.

[4] Li Xue. Researches on International Container Shipping Network Based on Complicated Network Betweenness Centrality. Dalian Maritime University, 2013, 41 (12): 123-124.

[5] Zhang Jiwei. Theory of Circle: Relations Promote the Development of Rural Credit Cooperative-Shanxi Linfen Yaodu District Rural Credit Cooperative. Shanxi Normal University,2013, 14 (41): 21-24. 
[6] Pan Feifei. Researches on Network Schedule Plan Standardization Based on Budget Quota of Hydropower Engineering. Tianjin University, 2010, 41(21): 22-24.

[7] Mr. Ashish Choubey,Ms. Latika Pande.A Practical approach for fault component network for Current and Voltage Phasor Diagram in Power Electronic Environment. International Journal of Advanced Computer Research,2012,1(2):21. 